\title{
Paper Using EEG Data and NeuCube for the Study of Transfer of Learning
}

\author{
Mojgan Hafezi Fard ${ }^{1}$, Krassie Petrova ${ }^{1}$, Maryam Doborjeh ${ }^{1}$, Nikola Kasabov ${ }^{1,2}$ \\ 1. School of Engineering, Computer and Mathematical Sciences, Auckland University of Technology \\ Auckland, New Zealand \\ 2. George Moore Chair of Data Analytics, Ulster University \\ mojgan.hafezi.fard@aut.ac.nz, krassie.petrova@aut.ac.nz,mgholami@aut.ac.nz,n.kasabov@aut.ac.nz
}

\begin{abstract}
Deeper and long-lasting learning occurs through a critical review of prior knowledge in the light of the new context, and a transfer of the acquired knowledge to new settings. Attention to task is one of factors that enable transfer of learning (TL). This study adopts a cognitive neuroscience approach to the study of TL; more specifically, to the investigation of the relationship between attention to task and prior knowledge. The study uses a Brain Like Artificial Intelligence (BLAI) architecture (NeuCube) which is based on Spiking Neural Networks (SNN) to represent brain data during a series of cognitive tasks and interpret them in the context of the research question. The experimental results indicate that modelling and analysing spatio-temporal brain data (STBD) using the SNN environment of NeuCube suggested a better understanding of the process of TL, and the associated brain activity patterns and relationships. The outcomes of this study are used to inform the design of a follow-up study where SNN models will be built from STBD gathered from participants engaged in learning and in TL.
\end{abstract}

Keywords- transfer of learning, attention, machine learning, spiking neural networks, NeuCube;

\section{INTRODUCTION}

Individuals learn when they develop an understanding of a concept to the extent of being able to apply it confidently in a different situation [1]. The ability to apply and adapt knowledge outside the context of learning is an important characteristic of deeper learning. It enables the development of " $21^{\text {st }}$ century skills" such as critical thinking and problem solving, and supports creativity and collaboration [2-3].

Deeper and long-lasting learning occurs through a critical review of prior knowledge in the light of the new context, and making connections between prior knowledge and the new knowledge gained [4]. Therefore, the ultimate education goal is to develop capabilities that allow individuals to both apply what was learnt, and extend, or transfer acquired knowledge to new settings [5].

The activation of prior knowledge and transfer of learning (TL) has attracted significant attention in both education and psychology research, as the goal of developing such capabilities is not always easily achieved [6]. However, there is still a lack of research findings that could be used to help learners transfer their knowledge or skills learned in one context, to a different one [3][7].
There is still a limited understanding of how previous knowledge and new information interact in the human brain [8]. Traditional data collection methods such as surveys and interviews have not provided sufficient insights into how TL occurs, due in part to the relatively low reliability of selfreported data which is subject to background bias (e.g., personal, socio-cultural) [9]. In addition, behavioural data cannot capture the subtle changes and differences in the cognitive process that underlies performance [10].

At the biophysical level, the human brain is the seat of learning [11-12]. Learning is a neurological phenomenon arising from physical changes in the brain cells. More specifically, the impact of learning on the brain is manifested by changes to the way neurons connect to each other thus changing the internal structure of the existing synapses. Therefore, a neuroscience approach to the study of TL may be more suitable and yield better results than a behavioural approach as it relies on objective rather than on subjective data related to the learning process. For example, the analysis of brain activity data collected from individuals engaged in learning may help to gain a deeper insight into how TL occurs and what factors may influence its success.

\section{A NEUROSCIENCE APPROACH TO THE STUDY OF TRANSFER OF LEARNING}

Recent advances in electroencephalography (EEG) have provided additional opportunities for studying the activities of the human brain in different contexts, including education [13-14]. The currently available low-cost, portable and easy to use EEG sensors can be set up to record functional brain wave measures in a learning context involving TL. The spatio-temporal brain data (STBD) generated by EEG devices can be used to build computational models, to enable the analysis and the interpretation of the emerging brain activity patterns [15].

\section{A. Spiking Neural Networks}

Advanced machine learning (ML) approaches such as neural networks can be used to study the inner working of the human brain, for example by exploring biological concepts such as working memory and attention [15]. The basic mechanism underlying learning and memory in biological neural networks is the ability of synaptic connections (connections between neurons) to change their strength, in order to communicate with each other (by 
generating and propagating electrical pulses, or spikes) [17]. Spiking neural networks (SNNs) mimic this behaviour of biological neural networks.

An STBD set collected through the use of an EEG, or a fMRI device can be fed into a SNN as a stream of spikes, in order to create a computational model of the brain activities represented by the dataset. SNNs have shown promising results in modelling the behavior of the brain and studying its learning potential [18]. Prior research has demonstrated that a particular SNN based architecture, the NeuCube, has been efficient in the analysis of STBD [13] [19-20].

\section{B. Attention to Task}

Any learning process involves TL as 'new' learning occurs in the context of existing knowledge. Learning can be seen as an information processing cycle that involves thinking, perceiving, processing the data, and transferring knowledge to new contexts. Yet, in educational settings, this process does not always occur in its entirely [21]; this may be one of the reasons behind the lack (inadequacy) of TL mentioned above.

Attention to the task is at the base of the learning process. It is closely linked to several functions performed by the working memory: encoding, maintenance and retrieval [22]. At the encoding stage, information gathered through perception is at the focus of attention. At the maintenance stage, the information is stored and protected in an active state while at the retrieval stage, the information is retrieved when it is attended to. Attention to the task is one of factors that enable TL. It has been suggested that TL depends on where attention is directed to, during the stages of the learning cycle [23].

The central question guiding this study is: How to investigate the relationship between prior knowledge and attention to task using a neuroscience approach? We apply the NeuCube computational architecture in order to represent the patterns underlying neural connectivity and spike activities of the brain occuring during a series of cognitive tasks, and interpret them in the context of the research question.

\section{METHODS}

NeuCube is a neuromorphic computational architecture, first introduced in [19] that can be used to create brain like artificial intelligence (BLAI) (see for example [15] [30]). It is a generic tool that can be applied across various domain areas for modelling, visualization, and classification of STBD. The three-dimensional SNN-based architecture of NeuCube recognizes spatio-temporal relationships "hidden" in the data to gain a deeper understanding of the phenomenon investigated.

For creating models out of spatio-temporal EEG data, the NeuCube architecture includes the following main modules: (1) input data encoding; (2) mapping the data into a 3D brain inspired SNN architecture; (3) unsupervised learning and model visualization; and (4) supervised learning and classification.

The input encoding module of NeuCube transforms the continuous STBD set into discrete spike sequences (trains) which are an indication of a signal change over time. Here, the threshold-based encoding (TBE) method is used. If the amplitude of the EEG signal change is reaching the pre-set firing threshold, the output is a spike. If the upward change exceeds the threshold, the spike generated is a positive one, and if the downward change reaches the threshold, then a negative spike is created; otherwise, there is no spike.

Applying a brain coordinate template such as Talairach coordinates, a 3D brain inspired SNNc is mapped (the SNN cube, or reservoir that contains the spatially located neurons). Then, the encoded spike sequences are transferred into the SNNc in the order of their timing. SNNc is first trained in an unsupervised mode on the spike sequences applying spike-timing dependent-plasticity (STDP) learning rule as a synaptic modification mechanism [24]. At the next stage, an evolving SNN (eSNN) output classifier such as a dynamic evolving SNN (deSNN), first introduced in [25], is trained in a supervised mode to learn and classify the spatiotemporal patterns represented by the spike sequences and their pre-defined classes.

\section{A. Dataset Description}

This study extracted and used data from an available dataset described in detail in [26-27]. The original dataset contained the EEG data collected from ten college students while they were watching two types of one-minute educational video clips on science and maths topics.

The video clips were of two pre-defined types: video clips that would be easily understood and not causing confusion as participants would be familiar with the subject area of the clip, and video clips which would be hard to understand and potentially confusing to participants as they would not have the necessary prior knowledge needed to understand the material presented. The classification was based on assumptions about the prior knowledge of the participants. A set of 20 videos was used, with 10 video clips of each type. Each participant watched ten randomly chosen video clips (five from each type). After each session, participants were asked to rate their perceptions of the video clip in term of how easy it was to understand it.

The EEG data were collected by use of a portable EEG monitoring device with a single channel EEG sensor which measures frontal brain lobe activity (International 10-20 scalp location: FP1). The raw data sample rate was $512 \mathrm{~Hz}$, with samples taken every 0.5 seconds. Overall, the dataset encompasses 100 data points, with each data point including 120+ samples. Represented as a MS Excel spreadsheet, the dataset consists of $12000+$ rows that span 15 columns including subject and video ID, self-reported video type, pre-defined video type, and 11 spatial features representing the EEG spectrum bands.

\section{B. Data Pre-Processing}

The dataset comprised 100 sample files which were extracted from the original dataset. Each sample contained the EEG data related to one participant watching one of the ten videos assigned to them. The samples were organized as matrices, with 112 rows of ordered time points (temporal features) and 11 columns of spatial features (Table I). 
TABLE I. SPATIAL FEATURES

\begin{tabular}{|l|l|l|}
\hline & \multicolumn{1}{|c|}{ Name } & \multicolumn{1}{c|}{ Description } \\
\hline 1 & Attention & $\begin{array}{l}\text { A measure of the level of the subject's mental } \\
\text { concentration (a proppriatry featuuire of the EEG } \\
\text { device used). }\end{array}$ \\
\hline 3 & Meditation & $\begin{array}{l}\text { A measure of the level of the subject's mental } \\
\text { relaxation (a proppriatry feature of the EEG } \\
\text { device used). }\end{array}$ \\
\hline 4 & Delta & The avcerage of the EEG data (rows 4-11 below) \\
\hline 5 & Theta & $4-8 \mathrm{~Hz}$ \\
\hline 6 & Alpha 1 & The lower part of the $8-12 \mathrm{~Hz}$ \\
\hline 7 & Alpha 2 & The higher part of the $8-12 \mathrm{~Hz}$ \\
\hline 8 & Beta 1 & The lower part of the $12-30 \mathrm{~Hz}$ \\
\hline 9 & Beta 2 & The higher part of the $12-30 \mathrm{~Hz}$ \\
\hline 10 & Gamma 1 & The lower part of the $30-100 \mathrm{~Hz}$ \\
\hline 11 & Gamma 2 & The higher part of the $30-100 \mathrm{~Hz}$ \\
\hline
\end{tabular}

As in the original dataset, the 100 samples were divided into two equal sized classes: 50 samples representing participants watching a video clip they found easy to understand, as they had sufficient prior knowledge in the subject area, and 50 samples representing participants watching a video clip they found hard to understand due to the lack of sufficient prior knowledge. Two class labels were created to reflect the two types of a cognitive task as reported by each subject: "insufficient prior knowledge" (class 1), and "sufficient prior knowledge" (class 2).

\section{RESULTS AND ANALYSIS}

\section{A. Parameter Optimisation and Classification Accuracy}

The set of samples was split in half so that $50 \%$ of the data were used for training, and the other $50 \%$ were used for testing the model. The NeuCube parameters were set as follows: The threshold-based representation (TBR) method was chosen for the encoding stage, with the spike threshold set to 0.5 [28]. Following also [29], at the unsupervised learning stage, the STDP learning rate was set to 0.01 , the potential leak rate was set to 0.002 , the firing threshold was set to 0.5 and the refractory time was set to $6 \mathrm{~ms}$. The accuracy achieved was $64 \%$. Subsequently, the model was evaluated by performing 5-fold, 10 -fold, and leave-one-out cross validation (LOOCV). The 10 -fold cross validation provided the highest accuracy result of $70.59 \%$

Next, we performed a model optimisation by applying an exhaustive grid search method to find the best values for a combination of three main parameters (STDP learning rate, drift and mod [29] in order to enhance the model accuracy. Every parameter was searched within a range, defined by a minimum and maximum value, through several iterations related to the number of steps for moving from minimum to maximum. The parameter setting was as follows:
- STDP learning rate: from 0.001 to 0.01 , step number: 5

- Drift: from 0.001 to 0.05 , step number: 5

- Mod: from 0.4 to 0.95 , step number: 5 .

After the parameter optimization, the overall classification accuracy reached by NeuCube was $85.76 \%$. For a comparison analysis, we used the same dataset and ran the classification experiment with several conventional ML methods, namely Support Vector Machine (SVM), Multilayer Perceptron (MLP) and Multivariable Logistic Regression (MLR). The achieved classification accuracy was significantly lower (under 30\%) than the NeuCube models. This was to be expected; the ML methods used have a limited ability to process data that are not static but temporally varied (continuous in time) while NeuCube is specifically designed for capturing spatio-temporal relationship in brain data such as EEG data [19].

\section{B. Neuron Connectivity}

NeuCube provides the means to visually represent the spatio-temporal interactions between the brain data features in a 3D SNN model and analyse their relationships over time [30]. After mapping the spatial components of EEG data to the SNNc, the neuron connectivity is initialised using the small-world connectivity. These connections are later modified based on the learning of incoming spike sequences in time during unsupervised learning which is performed using the STDP learning rule. In this study, we looked specifically at neuron connectivity, clustering and interaction in the trained SNNc to understand and interpret the significance of the input data features. Fig 1. shows the connections between the input neurons in the trained SNNc after the completion of the unsupervised learning stage. The colour of the neuron shows the strength of its activity: the brighter the colour, the stronger the activity.

The blue and red lines indicate positive (excitatory) and negative (inhibitory) connections between neurons, respectively. The thickness of the line reflects its weight value and implies the strength of the connection between two neurons. In other words, a 'thicker' connection line indicates a higher weight value, meaning a stronger impact of the neuron's firing on another neuron.

Fig. 2 shows how the connections between the neurons evolved throughout the training stages. At the start, the initial connections in the SNNc are sparse and generated randomly (Fig. 2a). During the training process, the spikes are fed into model in the order of their timing; this leads to modifying the connections around the input neurons.

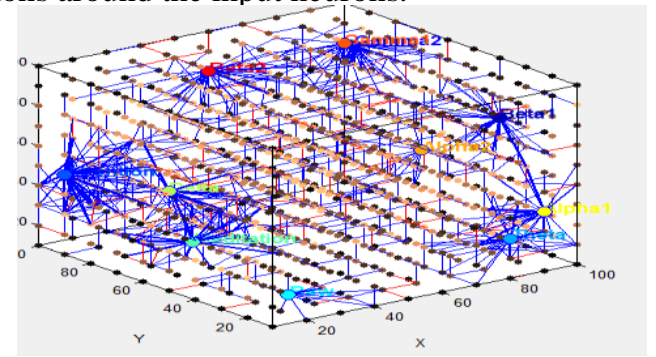

Figure 1. SNNc visualization: Neurons and connections between them 

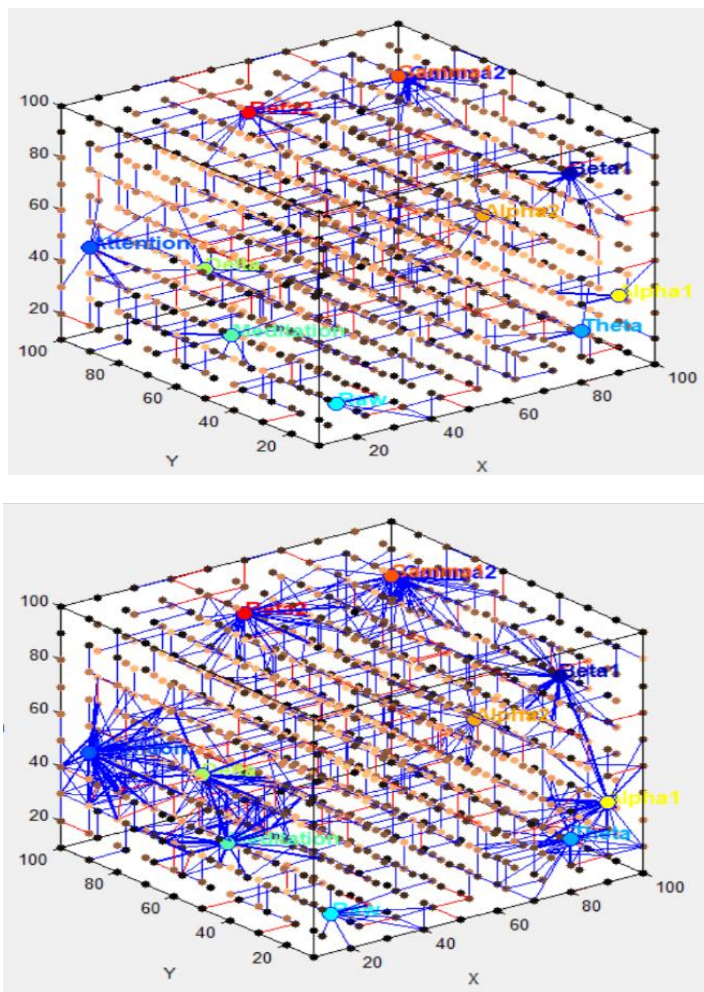

b)

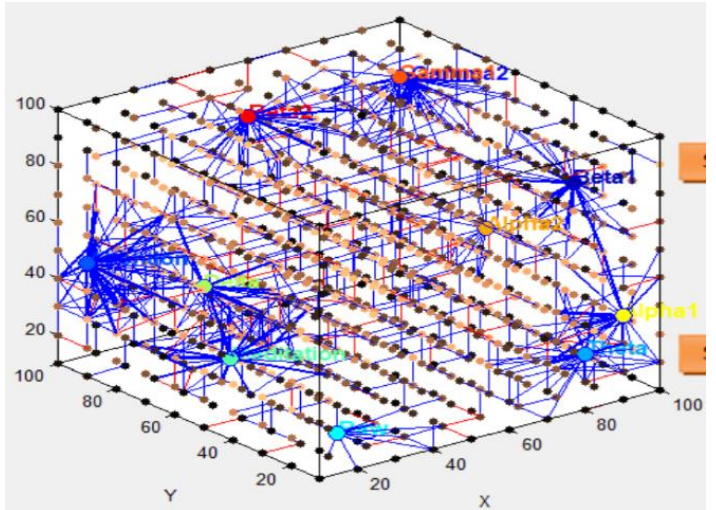

c)

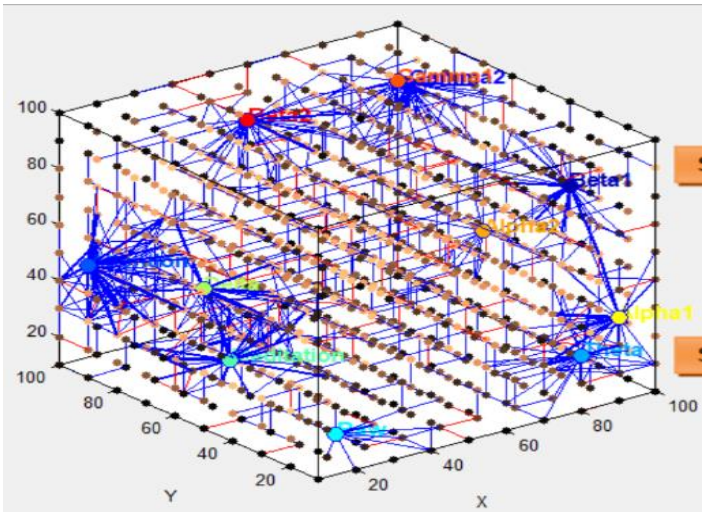

d)

Figure 2. SNNc visualization: (a) The initialised connections in the SNNc; (b-d) Evoloving neuronal connections throiughout the stages of training
In particular, the Delta input neuron shows strong interactions with two other input neurons (Attention and Meditation) (Fig 2b). As the process continues through to the last two training stages, the connections between each pair of neurons are getting stronger and at the same time, more connections are appearing (Fig. 2c-d). The emerging stronger neuronal connections in SNNc indicates that an increasing amount of temporal information (spikes) is exchanged between the neurons, with a higher level of learning activity occurring [30] [31].

\section{Connectivity Comparision}

For performing a connectivity comparison, the data samples for class 1 and class 2 were modelled separately in two SNN models. The visual representation of the neuronal connections in Fig. 3a-b, indicates that the connections in class 1 (insufficient prior knowledge) are stronger than the connections in class 2 (sufficient prior knowledge). The finding suggests that more information (spikes) was exchanged between the input neurons when a participant does not have prior knowledge related to the cognitive task compared to when a participant does have sufficient prior knowledge.

NeuCube also provides a new clustering method for visualising the level of spike communication between the neurons in the trained SNNc. It allows to better study the learning patterns during the STDP process.

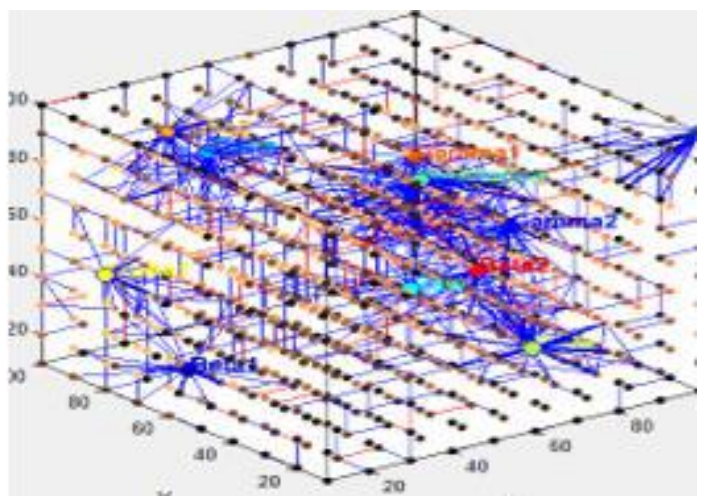

a)

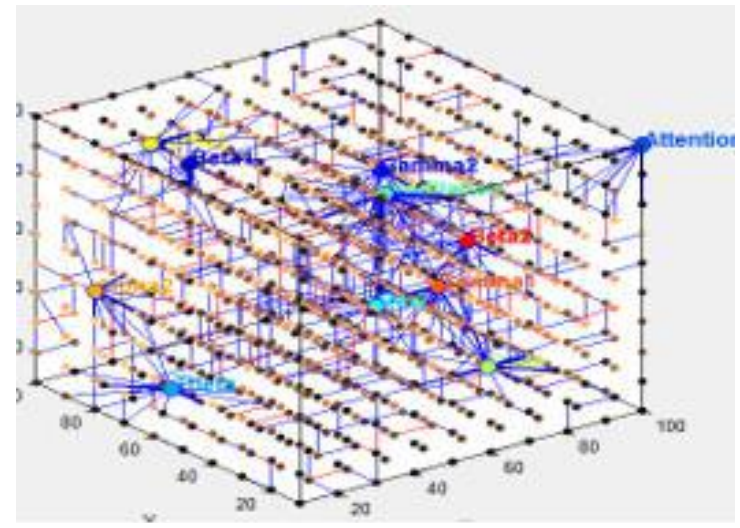

Figure 3. SNNc visualization: (a) connection strength for class 1 (insufficient prior knowledge; (b) connection strength for class 2 (sufficient prior knowledge). 
As neuronal connections evolve over time, neural clusters are formed around the input neurons; the cluster surrounding an input neuron contains the neurons that have received most spikes from this particular input neuron, compared to the rest of the input neurons [32]. In the trained SNNc model the size of a cluster surrounding an input neuron can be interpreted as an indication of the significance of the corresponding STBD input feature.

Normally, the cluster centres in a SNNc are predefined by the spatial locations of the input features (the EEG channels). However, in the dataset used in the study, the input features correspond to wave bands as the EEG data were gathered using only one EEG channel.

It can be seen in Fig. 4a that in class 1 (insufficient prior knowledge) the input neurons Attention and Beta 2 have the largest clusters therefore they are the most important features in class 1. In class 2 (sufficient prior knowledge, Fig 4b) the Attention sand Beta 2 clusters are relatively small; the three most important features are Theta, Meditation and Alpha 1, as their corresponding clusters are the largest. These three features are important in class 1 as well, as their respective clusters are also relatively large.

To gauge the relative strength of the interaction among neurons, we used the NeuCube's "neuron proportion" metric (the percentage of neurons in an input neuron cluster out of the total number of neurons in the SNNc). As seen in Fig. $5 a-b$, the neuron proportion of Attention in class 1 (insufficient prior knowledge) is $31 \%$ compared to $1 \%$ in class 2 (sufficient prior knowledge). There is also a difference between the neuron proportion of Alpha 1 (lower in class 1 compared to class 2 ).

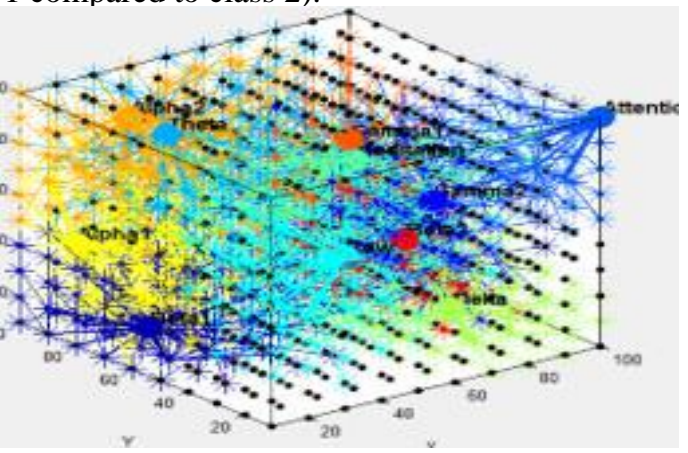

a)

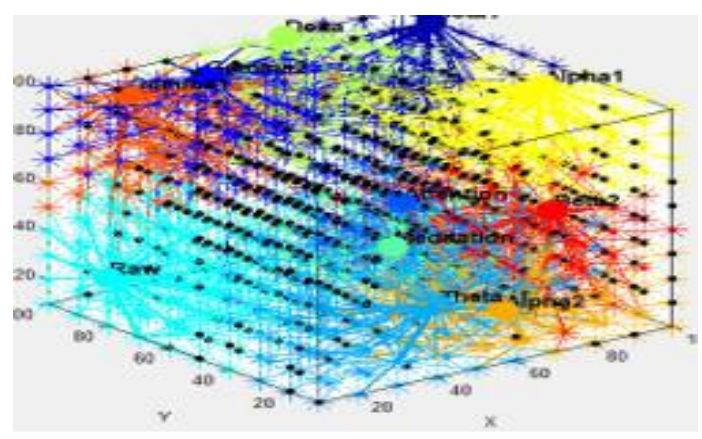

b)

Figure 4. SNNc visualization: (a) neuron clustering for class 1 (insufficient prior knowledge); (b) neuron clustering for class 2 (sufficient prior knowledge)

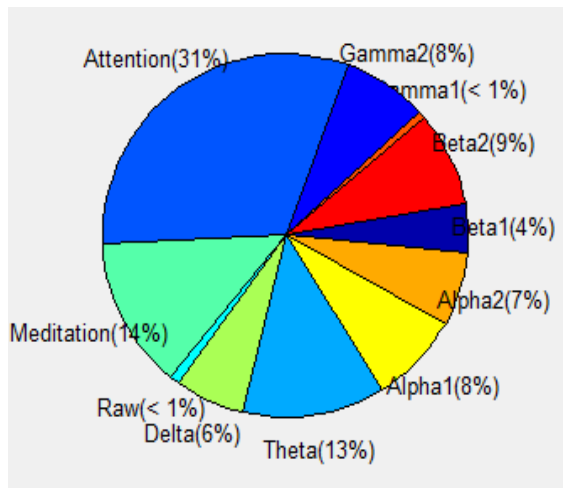

a)

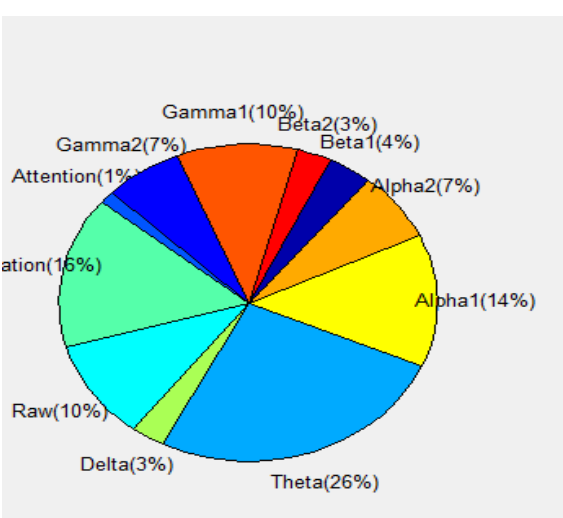

b)

Figure 5. SNNc visualization: (a) Neuron proprotion for class 1 (insufficient prior knowledge); (b) Neuron proportion for class 2 (sufficient prior knowledge)

\section{DISCUSSION}

The study aimed to investigate how a computational cognitive neuroscience approach could be applied to the study of TL, and in particular, the relationship between prior knowledge and attention to task. The experiment outcomes demonstrated that the classification accuracy of NeuCube model was sufficient and superior to the traditional ML methods.

Furthermore, the patterns emerging from the neuron cluster analysis and the neuronal interaction analysis showed plausible relationships between attention and prior knowledge as discussed below. First, the results of the analysis indicated that the size of the cluster and the amount of neuronal interaction associated with the Attention input neuron was significantly higher in the case of participants with insufficient prior knowledge (class 1). This may indicate that the intensity level of participants' concentration (attention) increases when they need to perform a cognitive task they are not prepared well for. So, when participants are not familiar with a task (as they do not have sufficient prior knowledge about the task), the attentional level increases accompanied by a decrease in the alpha band. However, when participants are familiar with the context of the cognitive task (i.e., they have the prior knowledge required in order to perform it successfully), attentional level to task decreases and the alpha band signal strength increases. The 
finding about the reverse correlation between attention to task and the alpha signal is in line with previous findings, for example, it is stated in [33] that a decrease in the alpha band is normally related to a drop in performance.

Second, the results indicated that the neuronal connections of the input neurons Theta and Meditation were stronger in class 2 (sufficient prior knowledge), suggesting that more information (spikes) was exchanged between the neurons in the respective clusters. The finding about the high neuronal activity in the Theta wave band and the high level of meditation are consistent with [34] where it is stated that meditation and Theta waves signify relaxed attention and a focus on inner experiences; more specifically, Theta activity is associated with memory recall . In class 1 (insufficient prior knowledge) the same neurons were still important but were receiving less information from their surroundings neurons and were less active in comparison to class 2 .

Finally, the finding about the higher neuronal communication related to the Beta 2 wave band in class 1 in comparison to class 2 is in line with [35] where the higher part of the Beta is found to correspond to active and anxious thinking occurring when a subject needs to maintain or increase their attention.

The experimental results indicate that modelling and analysing STBD using the SNN environment of NeuCube suggested a better understanding of the process of TL, and the associated brain activity patterns and relationships. This study contributes by demonstrating that the NeuCube SNN architecture can be used to build a model for the study of cognitive behaviour associated with TL.

\section{CONCLUSION}

To summarise, the NeuCube computational architecture was successfully applied to create a model which was used to identify and compare the patterns underlying neural connectivity and spike activities of the brain related to attention and the rest of the features in the input EEG dataset. In particular, the study used the EEG spectrum wave bands as spatial features to demonstrate the impact of prior knowledge on learner attention to the task; the results imply that if the attention of the learner is directed appropriately be selected external clues this can enhance the transfer of learning process.

The study has two major limitations. First, the EEG dataset used contained data gathered form one spatial channel. Thus, the resulting NeuCube model did not take full advantage of the spatio-temporal capability of NeuCube. Second, the cognitive task related to the dataset was not designed specifically for a TL scenario.

The limitations above will be addressed in the overarching (ongoing) research work that investigates how a deeper understanding of the brain activities associated with the TL process can help design learning and teaching environments that facilitate deeper learning. A further study is in progress where a methodology for the study of TL as a process contextualised through prior knowledge, is being developed. NeuCube and cognitive data analytics methods are used to build SNN models from multi-channel EEG data collected from participants both prior- and post a suitable learning experience. It is hoped that the analysis and interpretation of the emerging connectivity patterns will lead to a better understanding of how to stimulate TL in order to increase student engagement and achieve deeper learning.

\section{ACKNOWLEDGMENT}

Several implementations of the NeuCube architecture have been developed and made available free for study and research at www.kedri.aut.ac.nz/neucube and also at www.neucube.io, also funded by Auckland University of Technology (under the supervision of Prof. Nikola Kasabov) The MATLAB version was developed by a team from Auckland University of Technology including also Dr. Enmei Tu from Shanghai Jiao Tong University and the team of Prof. Hou from the CASIA Beijing. The cloud based and the open source versions were developed mainly by Dr. J. I. Espinosa Ramos and A. Ghollahalli from Auckland University of Technology.

\section{REFERENCES}

[1] T. McKelgan, "The disciplined mind. Howard Gardner". New York, NY: Simon and Schuster Inc. Brock Educ. J., vol. 9, no. 1, 1999.

[2] E. Rivière, M. Jaffrelot, J. Jouquan, and G. Chiniara, "Debriefing for the transfer of learning: The importance of context," Academic Medicine, vol. 94, no. 6, pp. 796-803, 2019.

[3] J.W. Pellegrino, and M.L. Hilton, "Education for life and work : developing transferable knowledge and skills in the 21 st century" , The National Academies Press, 2012.

[4] P. Mattews, "Near and far transfer of learning", Training Journal.com. https://www.trainingjournal.com/blog/near-and-far-transfer-learning. (Accessed Sep. 20, 2020).

[5] C. Bossard, G. Kermarrec, C. Buche and J. Tisseau, "Transfer of learning in virtual environments: a new challenge?," Virtual Reality, vol. 12 , no. 3, pp. 151-161, 2008.

[6] S. M. Barnett and S. J. Ceci, "When and where do we apply what we learn?: A taxonomy for far transfer," Psychological bulletin, vol. 128, no. 4 , p. 612,2002

[7] J. McTighe, and J. Willis, Upgrade your teaching: Understanding by design meets neuroscience, (ASCD, 2019).

[8] G. Brod, M. Werkle-Bergner and Y. Shing, "The influence of prior knowledge on memory: A developmental cognitive neuroscience Perspective", Frontiers in behav. neuroscience, vol. 7, 2013. Available: 10.3389/fnbeh.2013.00139.

[9] S. Lee et al., "Comparing programming language comprehension between novice and expert programmers using EEG analysis," IEEE 16th Int. Conf. Bioinf. BIBE. pp. 350-355, 2016

[10] J. Siegmund et al., "Understanding understanding source code with functional magnetic resonance imaging," Proc. 36th Int. Conf. Softw. Eng. , pp. 378-389, 2014.

[11] P. Mateos-Aparicio and A. Rodríguez-Moreno, "The impact of studying brain pasticity", Frontiers in Cellular Neuroscience, vol. 13, 2019.

[12] D. Michelon, "Brain Plasticity: How learning changes your brain", SharpBrains.com. https://sharpbrains.com/blog/2008/02/26/brainplasticity-how-learning-changes-your-brain/. (Accessed: Sep. 20, 2020).

[13] S. Doukakis, "Exploring brain activity and transforming knowledge in visual and textual programming using neuroeducation approaches", AIMS Neuroscience, vol. 6, no. 3, pp. 175-190, 2019. Available: 10.3934/neuroscience.2019.3.175.

[14] Crk, T. Kluthe and A. Stefik, "Understanding programming expertise:An empirical study of phasic brain wave changes", ACM Trans. Comput. Human Interaction, vol. 23, no. 1, pp. 1-29, 2015. 
[15] N. K. Kasabov, M. G. Doborjeh, and Z. G. Doborjeh, "Mapping, learning, visualization, classification, and understanding of fMRI data in the NeuCube evolving spatiotemporal data machine of spiking neural networks," IEEE Trans. Neural Netw. Learn. Syst, vol. 28, no. 4, pp. 887-899, 2017.

[16] R. C. Fong, W. J. Scheirer, and D. D. Cox, "Using human brain activity to guide machine learning," Scientific reports, vol. 8 , no. 1 , pp. 1-10, 2018.

[17] F. Ponulak and A. Kasinski, "Introduction to spiking neural networks: Information processing, learning and applications," Acta neurobiologiae experimentalis, vol. 71, no. 4, pp. 409-433, 2011.

[18] L. Lobo, J. Del Ser, A. Bifet and N. Kasabov, "Spiking neural networks and online learning: An overview and perspectives," Neural Netw., vol. 121, pp. 88-100, 2020.

[19] N. Kasabov, "NeuCube: A spiking neural network architecture for mapping, learning and understanding of spatio-temporal brain data", Neural Netw., vol. 52, pp. 62-76, 2014.

[20] E. Capecci, N. Kasabov, and G. Y. Wang, "Analysis of connectivity in NeuCube spiking neural network models trained on EEG data for the understanding of functional changes in the brain: A case study on opiate dependence treatment," Neural Netw., vol. 686, pp. 62-77, 2015.

[21] S. Forrester, "Transfer of learning and music understanding: A review of literature", Update: Applications of Research in Music Educ., vol. 37, no. 1, pp. 30-35, 2018

[22] J. Jonides et al., "The mind and brain of short-term memory," Annu. Rev. Psychol., vol. 59, pp. 193-224, 2008.

[23] J. R. Anderson, L. M. Reder, and H. A. Simon, "Situated learning and education," Educ. Researcher, vol. 25, no. 4, pp. 5-11, 1996.

[24] S. Song, K. D. Miller, and L. F. Abbott, "Competitive Hebbian learning through spike-timing-dependent synaptic plasticity," Nature Neuroscience vol. 3, pp. 919-926., Sept 2000.

[25] N. Kasabov, K. Dhoble, N. Nuntalid, and G. Indiveri, G,. “ Dynamic evolving spiking neural networks for on-line spatio-and spectrotemporal pattern recognition," Neural Netw., 41, pp.188-201, 2013.
[26] H. Wang et al.,'Using EEG to improve massive open online courses feedback interaction," vol. 1009, pp.59-66, 2013

[27] Z. Ni, A. C. Yuksel, X. Ni, M. I. Mandel, and L. Xie, "Confused or not confused?: Disentangling brain activity from eeg data using bidirectional lstm recurrent neural networks," ACM Proc. 8th Int Conf. on Bioinf., Comput. Biol., and Health Inform., 2017, pp. 241246.

[28] B. Petro, N. Kasabov, and R. M. Kiss, "Selection and optimization of temporal spike encoding methods for spiking neural networks," IEEE Trans. Neural Netw. Learn. Syst., vol. 31, pp. 358-370, April 2019.

[29] N. Kasabov and E. Capecci, "Spiking neural network methodology for modelling, classification and understanding of EEG spatiotemporal data measuring cognitive processes," Information Sciences, vol. 294, , pp. 565-575, 2015.

[30] N. K. Kasabov, Time-Space, Spiking Neural Networks and BrainInspired Artificial Intelligence, Heidelberg, Springer, 2019.

[31] E. Tu, N. Kasabov, and J. Yang, "Mapping temporal variables into the NeuCube for improved pattern recognition, predictive modeling, and undrstnding of stream data," IEEE, 2016.

[32] M. G. Doborjeh, N. Kasabov, and Z. G. Doborjeh, "Evolving, dynamic clustering of spatio/spectro-temporal data in 3D spiking neural network models and a case study on EEG data," J. Evolving Syst., vol. 9, no. 3, pp. 195-211, Jan. 2018.

[33] W.Klimesch, H. Schimke, and G. Pfurtscheller, "Alpha frequency, cognitive load and memory performance," BrainTopography, vol. 5, no. 3, pp. 241-251, 1993.

[34] J. Lagopoulos et al., "Increased theta and alpha EEG activity during nondirective meditation," J. Alternative and Complementary Medicine, vol. 15, no. 11, pp. 1187-1192, 2009.

[35] K. Takahashi, M. Saleh, R. D. Penn, and N. Hatsopoulos, "Propagating waves in human motor cortex," Frontiers in Human Neuroscience,vol. 5, p. 40, 2011. 